\title{
Ein neues System der Chalastogastra?
}

Von Fr. W. Konow in Teschendorf (Meklenburg).

Herr William H. A s h m e a d, Assistant-Curator am U.S. National-Museum in Washington, hat in The Canadian Entomologist, London 1898, pag. $141 \mathrm{ff}$,, ein neues System der „Chalastogastra" veröffentlicht, das einer kurzen Besprechung zu unterziehen ist. Der Herr Autor bezeichnet mit Recht die in Rede stehende Abtheilung der Hymenopteren als "Sub-Order"; aber wenn derselbe für diese Unterordnung den alten Namen „Phytophaga“ wählt, so müssen wir von Anf̣ang widersprechen, denn abgesehen davon, dass die entwickelten Insecten durchaus nicht phytophag, sondern wenigstens grossentheils carnivor sind, gibt es unter den übrigen Hymenopteren eine grosse Zahl, die im Larvenzustande gleichfalls auf Pflanzennahrung angewiesen ist. Es erscheint also völlig willkürlich, den obigen Namen lediglich auf die "Chalastogastra" beschränken zu wollen. Ueberdies hat bereits $\mathrm{L}$ a $\mathrm{m}$ a $\mathrm{r} \mathrm{k}$ denselben Namen bei den Mollusken verwandt. Dann greift Mr. A s h m e a d auf die H a r t i g'sche Zweitheilung in "Holz- und Blattwespen" zurück, indem er zwei "Series" annimmt, die er "Xylophaga" und "Phyllophaga" nennt. Auch hier müssen wir aufs entschiedenste widersprechen. $\mathrm{Zu}$ den "Xylophaga“ werden die Cephinen gerechnet, offenbar weil es noch bedenklicher wäre, dieselben als phyllophag zu bezeichnen; aber nnter den Cephinen kann doch nur ein kleiner Theil wie Janus und zur Noth allenfalls auch Syrista und Macrocephus als xylophag gelten, während sonst die ganze Tribus mit Recht den Namen "Halmwespen" trägt und unter den "Phyllophaga" dürften wenigstens die Gallenbewohner kaum als phyllophag bezeichnet werden können. Vor allen Dingen aber erscheint es völlig unmöglich, die Lydini und Xyelini mit den Tenthredinidae vereinigen und dieselben als gleichwerthig mit den Abtheilungen der letzteren auf gleiche Stufe stellen zu wollen, wenn man 
der natürlichen Verwandtschaft Rechnung tragen und nicht etwa nach Gutdünken ein willkürliches „System" schaffen will. Die Lydiden sind durch ihren ganzen Körper- und Flügelbau mindestens ebenso weit, wenn nicht weiter, von den Tenthrediniden entfernt, als die Siriciden. Ueberdies erscheint es durchaus verwerflich, die charakteristischen Merkmale für die einzelnen Abtheilungen des Systems von den Imagines, die Namen dieser Abtheilungen aber von dem Larvenzustande herzunehmen.

Die „Phytophaga“ werden bei Mr. A s h m ead in $15 \mathrm{Fa}$ milien getheilt, wovon 4 Familien auf die "Xylophaga" und 11 auf die "Phytophaga" kommen, nämlich der Reihe nach: Oryssidae, Siricidae, Xiphydriidae, Cephidae, Xyelidae, Lydidae, Hylotomidae, Lophyridae, Perreyiidae, Pterygophoridae, Selandriidae, Nematidae, Dineuridae, Tenthredinidae, Cimbrcidae. Da werden also die Lophyrini in 3, die Tenthredinini gar in 4 „Families" zerspalten, die alle auf gleicher Höhe mit den Cimbicinen und Arginen stehen sollen; die "Hylotomidae" werden in die nächste Verwandtschaft der "Lydidae", die "Selandriidae" neben die "Pterygophoridae" und die "Cimbicidae" neben die "Tenthredinidae" gestellt; die letzteren werden von den "Selandriidae" durch zwei Familien, die "Nematidae" und "Dineuridae" getrennt, ja was noch mehr, von den "Selandriidae" werden die "Strongylogasterinae" getrennt und als "Subfamily" zu den "Tenthredinidae" gerechnet, während die "Blasticotominae" als "Subfamily" zu den "Selandriidae" gezählt werden. Da könnte man fast auf den Gedanken kommen, dass der Herr Autor ohne irgend welche Kenntnis der Naturobjecte sein System lediglich nach Büchern ausgearbeitet habe. Jedenfalls hat derselbe nie in seinem Leben eine wirkliche Dineura gesehen, sonst hätte er nicht den wunderlichen Einfall haben können, die "Dineuridae" als „Family" von den „Nematidae" zu trennen und den übrigen „Families" als gleichwerthig gegenüberzustellen. Ebenso ist die Unterbringung der Blasticotoma bei den "Selandriidae" nur möglich, wenn man das Thier nie gesehen hat. Körperbau und Flügelgeäder weisen dieser Gattung ohne Zweifel ihren Platz in der Nähe der Xyelini an. Gänzlich verfehlt ist es auch, wenn Mr. A s h me a d die "Strongylogasterinae" von den "Selandriidae" abtrennt und zu den "Tenthredinidae" rechnet. Derselbe würde bald von dieser Aufstellung zurückkommen, wenn er etwa verurtheilt würde, die C a m e r o n'schen Strongylogaster aus Central-Ame- 
rika in die eine oder die andere Abtheilung zu vertheilen. Wahrscheinlich aber kennt Mr. A s h me a d überhaupt keinen Strongylogaster, denn die nordamerikanischen angeblichen "Strongylogaster" dürften wenigstens grösserentheils in die Gattungen Taxonus, Poecilosoma u. s. w. gehören. Dazu kommt, dass der Herr Verfasser sich bei den statuirten Gegensätzen seines Systems vielfach durch ein „usually“ helfen muss, und dass man infolge der oft nicht präcisen Gegensätze viele Gattungen schwer oder nur nach Willkür, andere wie Cladius u. s. w. überhaupt nicht in diesem „System“ unterbringen kann. Ein System kann doch nur den Zweck haben, die Gegenstände zu ordnen; hier ist aber alles ohne erkennbare Ordnung durcheinander gewürfelt, wie man ein Spiel Karten durcheinander mischt.

Was die weitere Theilung betrifft, so werden die „Siricidae" in zwei "Subfamilies", Siricinae und Tremecinae, getheilt. $\mathrm{Zu}$ den ersteren zählen Sirex und Paururus, zu den letzteren Xeris, Teredonia und Tremex. Diese Theilung kann anerkannt werden, wenn die kleine Tribus überhaupt getheilt werden soll. Doch müssen dit Subtribus dann Siricides und Tremecides heissen. Wenn aber Mr. A s h m a d eine neue Paururus-Art aufstellt, so hätte sich derselbe erst unter den amerikanischen PaururusArten umsehen müssen, ob seine angebliche nov. spec. nicht mit einer bereits bekannten Art zusammenfällt. Die schwarzen Beine und dunklen Flügel weisen auf areolatus Cress. hin, dessen Männchen apicalis Kirby ist. Zwar soll die neue Art eine rothe Hinterleibsspitze besitzen, während areolatus $q$ einen ganz dunklen Hinterleib hat. Aber die blosse Färbung begründet keinen specifischen Unterschied; und wenn Mr. A shme a d keine bestimmten plastischen Unterscheidungsmerkmale anzugeben weiss, so kann seine angebliche Art höchstens als weibliche Varietät des $P$. areolatus Cress. gelten. Wahrscheinlicher aber dürfte Mr. Ashmead die Färbung der Beine nicht richtig beurtheilt haben; und sein "P. pinicolus" dürfte nichts anderes als ein nenes synonymum zu $P$. nigricornis $\mathrm{F}$. sein. Wer übrigens die lateinische Sprache gebrauchen will, muss dieselbe gelernt haben. Das Wort pinicola ist substantivum und kann darum nicht in einen unglücklichen "pinicolus" verändert werden. 
Bei den "Cephidae" wird eine neue Art beschrieben : der Cephus Graenicheri ㅇ. Derselbe scheint sich aber in nichts von C. cinctus Nort. (= occidentalis Riley et Marlat) zu unterscheiden, der in der Färbung veränderlich ist, tür den aber die grösserentheils gelben Schenkel charakteristisch sind. Wenn die gelbe Färbung am Hinterleib hie und da röthlich geworden ist, so indicirt das nicht eine neue Art, sondern lässt vermuthen, dass das einzige A s h m e d'sche Exemplar ein wenig durch Cyankali oder sonstwie verfärbt sein dürfte. Wollte der Herr Autor eine nene Species beschreiben, so musste er dieselbe mit $C$. cinctus Nort. genau vergleichen und durch plastische Unterscheidungsmerkmale die Berechtigung derselben nachweisen.

Die "Xiphydriidae" werden wieder in zwei "Subfamilies", die "Derecyrtinae" und "Xiphydriinae" zerspalten; aber ein Blick auf die Gattung Derecyrta lehrt sofort, dass hier das Fehlen des Radialnervs kaum generischen Werth hat. Ein solch einzelnes Merkmal kann unmöglich eine Gattung, geschweige denn eine höhere Kategorie begründen. Viel wichtigere Merkmale als Derecyrta trennen die Gattung Konowia von den übrigen Xiphydriini, aber eine Theilung in Tribus ist hier ganz überflüssig, zumal da es sich dabei nur darum handeln würde, eine einzelne Art von den übrigen abzutrennen.

Dasselbe gilt von der Theilung der "Xyelidae in die "subfamilies" "Macroxyelinae" und "Xyelinae". Um die kleine Gattung Xyela nicht in ihrer "Family" allein stehen zu lassen, gründet der Herr Autor eine neue Gattung : Manoxycla, die sich dadurch von Xyela unterscheiden soll, dass die beiden Radialnerven in die zweite Cubitalzelle münden. Aber bei dem äusserst zarten Flügelgeäder der Gattung Xyela ist die Lage der Quernerven sogar innerhalb der einzelnen Arten eine unbeständige, kann also unmöglich einen Grund zu generischer Trennung abgeben. Die Gattnng Manoxyela kann nicht anerkannt werden.

Bei den "Lidydae" werden meine sämmtlichen Untergattungen zu Gattungen erhoben und ausserdem eine neue Gattung Liolyda gegründet. Mit dem ersteren kann ich nicht einverstanden sein, denn die vorhandenen Unterschiede genügen nach meiner Ueberzeugung nicht zur Begründung von Gattungen und so wird auch die Liolyda Ashmead wohl nicht als Gattung anerkannt werden können. Dieselbe wird auf Lyda frontalis Westw. basirt, die demnach nicht eine echte Lyda, sondern ein Pam-

Wiener Entomologische Zeitung, XVII. Jahrg., X. Heft (31. December 1898). 
philius sein müsste. Aber $\mathrm{Mr}$. A s hm e a d dürfte das W e stw 0 o d'sche Exemplar ebenso wenig wie Mr. C r e s s on gesehen haben und Westwood bildet als frontalis eine Lyda ab rinit einem Supraapicalsporn an den Vordertibien. Die L. frontalis Westw. muss also so lange als echte Lyda gelten, bis nachgewiesen wird, dass eine solche Lyda nicht existirt, und wenn dieser Nachweis erbracht werden sollte, so würde die W e s tw o od'sche Species als Phantasiegebilde ad acta gelegt werden müssen.

Gelegentlich der Theilung seiner "Lydidae" in die "subfamilies" "Megalodontinae" und "Lydinae" zeiht mich Mr. A s hme a d des Irrthums, wenn ich den der Subcosta zugewandten Theil des Discıidalnerven als "Basis" desselben bezeichnete. Aber es ist noch lange nicht ausgemacht, ob wirklich, wie Mr. A s h m e a d meint, die Quernerven als durch Spaltung der unter ihnen liegenden Längsadern angesehen werden müssen, oder ob dieselben aus den über ihnen liegenden Längsadern originiren, oder endlich ob sie nicht etwa als selbständige Gebilde anzusehen sind. Die letztere Meinung scheint auch mir nicht annehmbar zu sein, aber für seine Behauptung, dass "the basal nervure in reality represents a fork of the median vein ${ }^{4}$ dürfte der Herr Autor ausser seiner ganz subjectiven Ueberzeugung irgendwelche Begründung beizubringen kaum im Stande sein. Wenn irgendwo im Flügelgeäder ein Quernerv fehlschlägt, so pflegt sich gewöhnlich eine Spur desselben nicht nur an der hinteren, sondern auch an der vorderen Längsader zu ffnden, eine Thatsache, die dafür sprechen könnte, dass beide Adern an der Bildung des Quernerven betheiligt sind; ich für meinen Theil schliesse daraus, dass den Quernerven eine gewisse Selbständigkeit zukommt, wenn auch eine Selbständigkeit secundärer Art. Aber wie will man die Thatsache erklären, dass etwa bei Cephaleia, Pamphilius u. s. w. der Brachialnerv nie am Medius, sondern stets am Brachius verkürzt ist, ohne an letzterem irgend eine Spur zu hinterlassen. Das ist nur möglich, wenn dieser Nerv vom Medius originirt; und was dem einen recht ist, ist dem andern billig; wir können nicht den einen Nerv aus der vorderen und den anderen aus der hinteren Ader ableiten. Ueberdies weiss jeder kundige Systematiker, dass der systematische Werth des einzelnen Organs im Flügelgeäder um so grösser zu sein pflegt, je näher dasselbe dem Costalrande 
des Vorderflügels liegt. Die Bildung des Flügelgeäders kann nicht als von hinten, sondern muss als von vorn ausgehend gedacht werden. Doch immerhin ist es nicht meine Meinung, dass wir mit unserer Terminologie an morphologische Begründung gebunden seien. Mag man also meinetwegen weiter von einer "furca medii, furca brachii“ u. s. w. reden und wenn es Gebrauch bei den meisten Fachgelehrten ist, bin auch ich gerne bereit, die Basis des Discoidalnerven am Medius zu suchen.

Ueber die von Mr. A s h m e a d für die "Hylotomidae" gegebenen Tabelien zu urtheilen bin ich zur Zeit nicht im Stande, weil mir mehrere hieher gehörige Formen fehlen und weil der Autor bei den meisten Gattungen typische Species anzugeben unterlässt. Deswegen nur einige Bemerkungen. Die Gattung Pachylota wird bei den "Schizocerinae" aufgeführt, gehört aber zu den Argides. Ferner wird dreimal eine Gattung "Sihizocera" Latreille aufgeführt, die theils drei, theils vier Cubitalzellen, theils ein contrahirtes, theils ein petiolirtes Humeralfeld im Vorderflügel besitzen soll. Nun hat L a t r e i ll e meines Wissens überhaupt keine Gattung "Schizocera" aufgestellt, auch wohl kaum irgend eine hieher gehörige Species gekannt. Der Name Schizocera stammt von Lepeletier und wurde 1829 von Curtis in "Schizocerus" verändert, muss aber nothwendig Schizoceros lauten, da nur diese Form sprachlichen Regeln entspricht. Es ist aber sehr fraglich, ob dieser Gattungsname überhaupt für die Gattung anwendbar ist, die gegenwärtig damit bezeichnet wird. Als Lepeletier 1825 in Encyclop. méthod. vol. 10 pag. 572 die Gattung Schizoceros gründete, kannte er vier oder vielmehr nur drei Arten, die er in folgender Reihenfolge aufzählt: 1. S. furcata, 2. S. Klugi, 3. S. pallipes, und 4. S. angelicae. Davon ist Nr. 2 identisch mit Hylotoma plumigera Kl.; Nr. 3 ist $=$ Tenthredo (Cyphona) geminata Gmel.; und Nr. 4 ist eine weibliche Varietät der Tenthredo (Cyphona) furcata Villers. Leider ist mir die nordamerikanische plumigera Kl. nicht bekannt, so dass ich nicht beurtheilen kann, ob dieselbe wirklich von den übrigen Lepeletier'schen Schizoceros-Arten generisch verschieden ist und dann als Typus der Gattung Schizoceros angesehen werden muss, oder ob der L e p e le t i e r'sche Name etwa einfach statt des Fallen'schen Cyphona einzutreten hat.

Die Gattung Atomaceros Say, die für die nordamerikanischen Arten debilis say und cellularis Say gegründet wurde,

Wiener Entomologische Zeitung, XVII. Jahrg., X. Heft (31. December 1898). 
wird von Mr. A s hme a d eingezogen und beide Arten werden zu Schizoceros gerechnet, während für Atomaceros ruficollis Nort. eine neue Gattung, Micrarge, aufgestellt wird, die zu den Argides gehören soll. Aber ein Argide ohne Anhangszelle im Vorderflügel und mit gegabelten Fühlern beim $\sigma^{7}$ ist sehr auffällig und überdies soll nach $W$ alsh der ruficollis Nort. das Weibchen von $A$. debilis Say sein. Wenn die letztere Vermuthung sich bestätigen sollte, so würde gerade für ruficollis Nort. der $\mathrm{S}$ a y'sche Gattungsname Atomaceros bleiben müssen. Dass übrigens Thiere mit gestieltem und solche mit contrahirtem Humeralfelde nicht in einer Gattung vereinigt bleiben können, habe ich schon früher ausgeführt.

Als Gymnia Spin. gelten bisher nach K i r b y diejenigen Arten, bei denen im Vorderflügel die Anhangszelle fehlt und von den drei Cubitalzellen die erste und zweite je einen Medialnerven aufnehmen. Nach Mr. As hmead soll Gymnia im Vorderflügel eine Anhangszelle besitzen, während die ersteren Arten zu Schizoceros gerechnet werden. Mir sind leider die typischen Arten apicalis und tibialis unbekannt. Beiläufig aber sei auch hier bemerkt, dass wir in der Entomologie als Gattungsnamen nicht irgendwelche aus einer $x$-beliebigen Sprache entnommenen Worte oder wortähnliche Formen gebrauchen können, sondern die Gattungsnamen müssen lateinisch sein. Deswegen sind Formen wie Themos, Ptenos, Acanthoptenos unmöglich; es muss Themus, Ptenus u. s. w. heissen. Ferner ist zu schreiben: Sericoceros und Dieloceros. Was den Namen Themus betrifft,

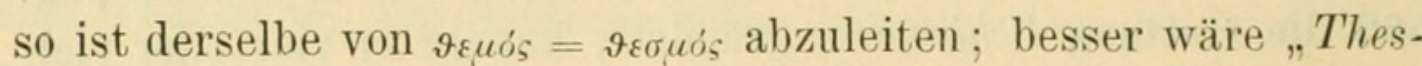

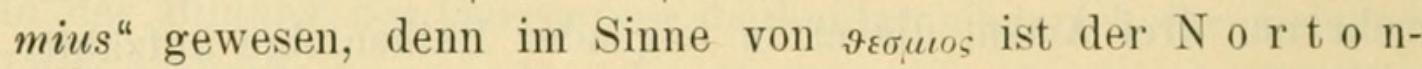
sche Name wahrscheinlich gedacht.

Die Unterfamilie der Lopliyrini habe ich erst kürzlich in den Entom. Nachrichten 1898, pag. $247 \mathrm{ft}$. auseinandergesetzt. Mr. A s h mea d zerreisst diese Unterfamilie in drei selbständige Familien, die Lophyridae, Perreyiidae und Pterygophoridae; und die letzte "family" wird in drei „subfamilies" getheilt, nämlich in die Lobocerinae, Pterygophorinae und - Perginae. Das ist also ein schönes Sammelsurium, das hier in der letzten „family" wegen des einzigen Merkmals der fehlenden lanzettförmigen Zelle vereinigt ist. Es ist für den Systematiker ja äusserst bequem, nach willkürlich herausgegriffenen augenfälligen Merkmalen sein System aufzubauen; nur schade, dass 
die Natur sich absolut nicht nach künstlichen Gesetzen richten will. Mr. A s h m e a d wird für diese Aufstellungen bei ernsthaften Entomologen kaum Zustimmung finden. Nicht einmal auf L i n n é wird sich derselbe für seine Kunst berufen dürfen, denn der alte Linné hat nach einheitlichem Princip und darum ein wirkliches, wenn auch künstliches, System aufgestellt.

Bei den "Perreyiidae" scheint die Tabelle zur Bestimmung der Gattungen wieder ohne Kenntniss der Naturobjecte zusammengestellt zu sein; sonst hätte der Herr Verfasser sehen müssen, dass hier weder die Zahl der Fühlerglieder, noch die Länge oder Kürze der zweiten Cubitalzelle, oder die Lage der Quernerven, wahrscheinlich auch nicht einmal die Zahl der Palpenglieder sichere Merkmale zur Unterscheidung von Gattungen darbieten. Wenigstens die Zahl der Fühlerglieder und die Lage der Quernerven ist oft nicht einmal bei derselben Art constant. Uebrigens soll die Gattung Perreyia nach $\mathrm{C}$ a m er o n 13-, nach A s hm ea d 15 gliederige, die Gattung Decameria nach älteren Autoren 10-, nach A s h m e a d 11 gliederige Fühler besitzen. Die Perreyia-Exemplare meiner Sammlung haben 13- bis 18 gliederige und meine Decameria - Arten 10 gliederige Fühler. Die Gattung Lophyruides Cameron ist vielleicht von Perreyia generisch verschieden, muss dann aber besser begründet werden, als es vom Autor geschieht. Dieselbe wird hauptsächlich auf die mehr als 13 gliederigen Fühler begründet, die beim $\sigma^{\top}$ flabellirt sein sollen. Leider ist mir nur ein einziges Perreyia-Männchen bekannt, das 14 einfache Fühlerglieder ohne Kammstrahlen besitzt. Immerhin ist zu vermuthen, dass diejenigen Arten, deren Männchen fiederstrahlige Fühler besitzen, einer anderen Gattung angehören dürften. Aber hier entsteht eine Schwierigkeit: Mr. Cam e r o n stellt als erste Art seiner Gattung Lophyroides den Lophyrus tropicus Nort. hin; dieser aber besitzt nach N o r t o n's Gattungsdiagnose an jedem Fühler 2 Reihen Fiederstrahlen, während $\mathrm{C}$ a m e ron die Fühler einfach "flabellate" nennt. Es scheint also, dass C a m e r o n's Lophyroides tropicus nur eine Reihe Fiederstrahlen an seinen Fühlern hat; und wenn das der Fall sein sollte, so würde der L. tropicus Cam. einfach als Synonym zu L. anomalus Kirby zu stellen sein, während der L. tropicus Nort. noch der Deutung harren müsste. Jedenfalls ist die Aufstellung des Mr. A shmead durchaus nicht annehmbar. Der letztere will auf 
den L. tropicus Nort. die neue Gattung "Lophyridea" gründen, obwohl er den L. tropicus Nort. wahrscheinlich gar nicht kennt. Seine Gattung soll sich von Lophyroides dadurch unterscheiden, dass die Hinterflügel eine geschlossene Cubitalzelle und am Radialfelde keine Anhangszelle besitzen. Das letztere könnte auffallen. Aber Cameron's L. tropicus hat offenbar eine Anhangszelle im Hinterflügel; und wenn Mr. A s h mead ein Exemplar ohne solche Zelle gesehen hat, so dürfte es sich da um ein zufälliges Fehlschlagen desjenigen Theiles der Randader handeln, der sonst das Radialféld schliesst, wie es auch bei anderen Arten vorkommt. Die Hinterflügel sind gewöhnlich wenig geeignet, Gattungsmerkmale herzugeben; und zumal bei der Gattung Perreyia ist das Flügelgeäder überhaupt höchst unbeständig. Die Gattung Lophyridea Ashmead kann nicht anerkannt werden.

Wenn Mr. A s hm e d die Gattungen Incalia, Paralypia, Cerealces statt bei den Cimbiciden vielmehr bei den "Lobocerinae" (Lobocerotides) unterbringt, so ist das durchaus zu billigen; aber eben damit fallen auch die hauptsächlichen Merkmale, die der Herr Autor für seine "Lobocerinae" beibringt, völlig in sich zusammen. Dass das "costal vein slender towards base but very much thickened or broadened at apical one- third" sei, trifft höchstens bei Acorduleceros zu; und ein „marginal cell not appendiculated findet sich nur wenig bei Lobocerotides! Gleichwohl meint der Herr Autor mit diesen Angaben die Schwierigkeiten „überbergt“ zu haben, die sich ihm bei Auseinandersetzung seiner "subfamilies" entgegenstellten. Derselbe hätte entschieden besser gethan, sein ganzes System im Papierkorb zu begraben, als es an die Oeffentlichkeit zu bringen.

Die "Pterygophorinae" sind bei Mr. A s h m e a d ein buntes Gemisch aus den verschiedenartigsten Formen. Thulea, Pterygophorus, Brachytoma, Syzygonia, Corynophilus und wieder Pterygophorus stehen ganz wirr durcheinander. Aber auch neue Gattungen gründet Mr. A s h m e a d hier : „Lophyrotoma" und "Pterygophorinus" werden von Pterygophorus abgetrennt. Der letztere soll 4 Cubitalzellen besitzen und wird auf $P$. cinctus beschränkt, während bei den ersteren, deren Typen $P$. interruptus und analis sind, nur 3 Cubitalzellen vorhanden sein sollen. Die ganze Sache ist Blech. Klug hat sicher besser zählen können als Mr. A s h m ead, der sich auch sonst gern verzählt; und $\mathrm{Klug}$ 
zählt bei $P$. cinctus nur 3 Cubitalzellen. Ferner soll $P$. analis Costa nach dem Autor 17-, nach A s h m e a d 23 gliederige Fühler haben; ein Exemplar, das ich für analis Costa halte, besitzt 19 Fühlerglieder. Ein ernsthafter Entomologe kann die $P$. cinctus, interruptus und analis unmöglich generisch auseinanderreissen. Uebrigens ist der schneidige Name Lophyrotoma ganz unverständlich. Derselbe kann nur aus Lophyrus und tźavєı gebildet sein; und man weiss nicht, was denn hier eigentlich geschnitten sein soll; wahrscheinlich hat sich der Herr Autor selbst mit diesem Namen schneiden wollen. Ebenso wird von Syzygonia die neue Gattung "Syzygonidea" abgetrennt. Die erstere soll 5-jointed, die letztere soll 6-jointed Fühler haben; aber Syzygonic Kl. hat nach $\mathrm{Klug}$ selbst schon vor der Fühlerkeule 5 Glieder; und die Keule ist wenigstens aus 3 bis 4 Gliedern zusammengewachsen. Ausserdem soll Syzygonia im Hinterflügel eine geschlossene Medialzelle besitzen, während Syzygonidea eine solche entbehren soll. Mir ist, nur die S. cyanoptera Kl. bekannt; un? diese hat vor der Fühlerkeule 5 Glieder und in Hinterflügel nur eine geschlossene Cubitalzelle, müsste also nach Mr. A s hmead eine dritte Gattung bilden, wofür demselben vielleicht die Namen "Syzygonides" oder "Syzygoniotoma" vorgeschlagen werden könnten!

Die Gattung Brachytoma Westwood, die von Cameron mit Perreyia vereinigt wurde, wird von Mr. A s h mead nicht nur wieder als selbständig aufgenommen, sondern ganz aus der Verwandtschaft der Perreyia herausgerissen und zu den "Pterygophorinae" gebracht. Typus der Gattung soll B. fumipennis Westw. sein, ein Männchen, welches nach Westwood zweistrahlige Fühler besitzt. Wenn nun dies Männchen wirklich, wie es nach A s h me ad scheint, das Humeralfeld im Vorderflügel entbehren sollte, so ist damit noch lange nicht bewiesen, dass es zu den Pterygophorides gehört; aber wahrscheinlich ist bei dem Exemplar des Herrn Ash mead nur der Humerus zufällig fehlgeschlagen.

Von der Gattung Perga werden drei neue Gattungen abgezweigt, nämlich: Paraperga, Pseudoperga und Neoperga. Die erste und letzte können möglicherweise gehalten werden. Die zweite wird auf $P$. polita Leach gegründet und soll 7 gliederige Fühler und nur 3 Cubitalzellen besitzen. Aber $P$. polita Leach hat sowohl nach dem Autor als auch nach K i r b y 6 gliederige

Wiener Entomologische Zeitung, XVII. Jahrg., X. Heft (31. December 1898). 
Fühler, wenn nämlich die Fühlerkeule als ein Glied gezählt wird, was allerdings mit Unrecht geschehen dürfte; und ausserdem hat $P$. polita gar nicht 3 , sondern 4 Cubitalzellen ; der erste Cubitalnerv ist nur mehr weniger bleich, weswegen Le a ch sagt: "areolis submarginalibus 2 primis confuentibus". Jedenfalls dürfte es völlig unmöglich erscheinen, die $P$. polita Leach von $P$. Kirbyi Westw. generisch zu trennen. Ueberdies hat bereits $1845 \mathrm{G}$ u ér in in Iconogr. règn. anim. vol. 7, pag. 398 ein Genus Pseudoperga gegründet und dasselbe auf $P$. Lewisi Westw. und ventralis Guér. basirt, die gleichfalls 3 Cubitalzellen aber 6 gliederige Fühler haben sollen. Mr. As h m e a d kommt also mindestens zu spät.

Derartige Aufstellungen fördern nicht, wie Mr. A s hme a d sich schmeichelt, das Studium der in Rede stehenden Thierchen, sondern sind höchstens geeignet, den, der diese Tabellen benützt, zu verwirren und in die Irre zu führen. 


\section{$2 \mathrm{BHL}$ Biodiversity Heritage Library}

Konow, Friedrich Wilhelm. 1898. "Ein neues System der Chalastogastra?"

Wiener entomologische Zeitung 17, 268-278.

https://doi.org/10.5962/bhl.part.3135.

View This Item Online: https://www.biodiversitylibrary.org/item/44201

DOI: https://doi.org/10.5962/bhl.part.3135

Permalink: https://www.biodiversitylibrary.org/partpdf/3135

\section{Holding Institution}

Smithsonian Libraries

\section{Sponsored by}

Smithsonian

\section{Copyright \& Reuse}

Copyright Status: NOT_IN_COPYRIGHT

This document was created from content at the Biodiversity Heritage Library, the world's largest open access digital library for biodiversity literature and archives. Visit BHL at https://www.biodiversitylibrary.org. 